\title{
Spheres from cervical cancer cells display stemness and cancer drug resistance
}

\author{
HUAN LIU $^{1 *}$, HAIJUAN WANG ${ }^{*}$, CHUNXIAO LI $^{1}$, TINGTING ZHANG ${ }^{2}$, XITING MENG $^{1}$, \\ YING ZHANG $^{3}$ and HAILI QIAN ${ }^{1}$
}

\author{
${ }^{1}$ State Key Laboratory of Molecular Oncology, Cancer Institute/Hospital, \\ Peking Union Medical College and Chinese Academy of Medical Sciences, Beijing 100021; ${ }^{2}$ Guangdong Medical College, \\ Dongguan, Guangdong 150080; ${ }^{3}$ Department of Gynecology, Minimally Invasive Center, \\ Beijing Obstetrics and Gynecology Hospital, Capital Medical University, Beijing 100006, P.R. China
}

Received January 26, 2015; Accepted February 19, 2016

DOI: $10.3892 / 01.2016 .4893$

\begin{abstract}
Cervical cancer is one of the most common gynecological malignant tumors and is the cause of a serious health problem worldwide. An increasing amount of evidence has shown that cancer stem cells (CSCs) are present in tumors, and that these CSCs may be responsible for tumor metastasis and relapse. The present study aimed to identify and characterize a CSC population from the CaSki cell line. First, a stem cell culture medium was used to selectively expand the cancer stem-like cell spheres, and the putative stemness markers, Oct 4 and Sox2, were identified. These markers were all highly expressed in the CaSki sphere-forming cells. Next, target region amplified polymorphism-polymerase chain reaction was performed and the CaSki sphere-forming cells were found to exhibit higher telomerase activity than the CaSki control cells cultured in non-stem cell medium. Using the 3-(4,5-dimethylthiazol-2-yl)-5-(3-carboxymethoxyphenyl)-2-

(4-sulfophenyl)-2H-tetrazolium assay, it was found that the CaSki sphere-forming cells were more resistant to chemotherapeutic drugs than the control CaSki cells. Using the tumor invasive assay, it was shown that the CaSki sphere-forming cells were more invasive than the control CaSki cells. These characteristics all suggested that the tumor sphere-forming
\end{abstract}

Correspondence to: Professor Haili Qian, State Key Laboratory of Molecular Oncology, Cancer Institute/Hospital, Peking Union Medical College and Chinese Academy of Medical Sciences, 9 Dongdan San Tiao, Beijing 100021, P.R. China

E-mail: qianhaili001@163.com

Dr Ying Zhang, Department of Gynecology, Minimally Invasive Center, Beijing Obstetrics and Gynecology Hospital, Capital Medical University, 17 Qi He Lou Street, Beijing 100006, P.R. China E-mail: zyandzm@163.com

*Contributed equally

Key words: cancer stem cell, cervical cancer, cancer metastasis, apoptosis, drug resistance cells mirrored the acknowledged CSC phenotypes. Overall, the use of a suspended sphere culture of CaSki cells may be an easy and feasible approach for enriching cancer stem-like cells in cervical cancer research.

\section{Introduction}

Cervical cancer is the fourth most common cancer in women following breast, lung and colorectal cancer with an incidence rate of $6 \%$ (1), and it is one of the leading causes of cancer-related mortality in women with a mortality rate of $3 \%$ in the USA (2). Significant progress has been achieved in cervical cancer treatment with the emergence of novel therapeutic drugs and treatment approaches (3). However, for patients in the advanced stages of disease, tumor relapse and resistance to radiation and available therapeutic approaches is inevitable. Therefore, investigation of the mechanisms of tumor relapse and drug resistance is a matter of great urgency.

In recent years, the cancer stem cell (CSC) theory has become widely accepted; it was found that CSCs, which represent a small fraction of tumor cells in the bulk of the tumor, are characterized by self-renewal, infinite proliferation and multiple differentiation potentials, and may be the root cause of tumor relapse and drug resistance $(4,5)$. At present, the sorting methods of CSCs include flow cytometry based on special cell surface markers, the side population cell sorting technique, tumor sphere culturing and the ALDEFLUOR ${ }^{\mathrm{TM}}$ assay (6-9). Previous studies have identified the CSCs from colon, breast and glioblastoma cancer, as well as other cancer cell lines, using the sphere culturing method (10-12). Tumor cell sphere culturing may be a convenient method for generating cancer stem-like cells to be used in the research of malignant behavior.

The present study attempted to identify a population with CSC properties from the CaSki cell line and performed a preliminary evaluation of the role of CSCs in tumor relapse and drug resistance.

\section{Materials and methods}

Cell lines and cell culture. The human cervical carcinoma epithelioid CaSki cell line was maintained in the State Key 
Laboratory of Molecular Oncology, Cancer Institute/Hospital, Peking Union Medical College and Chinese Academy of Medical Sciences (Beijing, China), and were purchased from Cell Resource Center, Institute of Basic Medical Science, Peking Union Medical College, Chinese Academy of Medical Sciences (Beijing, China). The cells were routinely grown in Dulbecco's modified Eagle's medium (DMEM) supplemented with $10 \%$ fetal bovine serum, penicillin $(100 \mathrm{U} / \mathrm{ml})$ and streptomycin $(100 \mathrm{mg} / \mathrm{ml})$ at $37^{\circ} \mathrm{C}$ in a $5 \% \mathrm{CO}_{2}$ standard incubator.

Antibodies. The following antibodies were used: Anti-SRY-box 2 (Sox2) rabbit anti-mouse polyclonal antibody (1:1,000 dilution; catalogue no. ab59776; Abcam, Cambridge, UK), anti-POU class 5 homeobox 1 (Oct4) rabbit anti-mouse polyclonal antibody (1:1,000 dilution; catalogue no. ab18976; Abcam), anti- $\beta$-actin mouse anti-rabbit monoclonal antibody (1:5,000 dilution; catalogue no. A5316; Sigma-Aldrich, St. Louis, MO, USA), anti-ATP binding cassette subfamily G member 2 (ABCG2) rabbit anti-human polyclonal antibody (1:1,000 dilution; catalogue no. BS3482; Bioworld Technology Inc., Nanjing, China) and anti-poly (ADP-ribose) polymerase (PARP) rabbit anti-human polyclonal antibody (1:1,000 dilution; catalogue no. ab6079; Abcam).

Tumor sphere culturing. The CaSki cells were cultured in an incubator at $37^{\circ} \mathrm{C}$ with $5 \% \mathrm{CO}_{2}$, and were digested into single cells suspension and replanted into 6 -well plates (500 cells/well) in serum-free DMEM-F12 (Gibco; Thermo Fisher Scientific Inc., Waltham, MA, USA), supplemented with $10 \mathrm{ng} / \mathrm{ml}$ basic fibroblast growth factor (PeproTech Inc., Rocky Hill, NJ, USA), $20 \mathrm{ng} / \mathrm{ml}$ epidermal growth factor (PeproTech Inc.), $25 \mathrm{ng} / \mathrm{ml}$ insulin (Novo Nordisk, Bagsværd, Denmark), $4 \mu \mathrm{g} / \mathrm{ml}$ heparin sodium (Changzhou Qianhong Bio-Pharma Co., Ltd., Changzhou, China), $20 \mathrm{nM} / 1$ progestin (Amresco, Solon, OH, USA), $30 \mathrm{nM} / 1$ sodium selenite (Amresco), $100 \mu \mathrm{g} / \mathrm{ml}$ apo-Transferrin (Sigma-Aldrich), $4 \mathrm{mg} / \mathrm{ml}$ bovine serum albumin (Sigma-Aldrich), $4 \mathrm{mg} / \mathrm{ml}$ glucose (Amresco) and $2 \mathrm{mM} / 1$ L-glutamine (Amresco). After 5-7 days, the tumor spheres were collected by centrifugation at $157 \mathrm{x}$ g, digested with Accutase (Sigma-Aldrich) to generate single cells and passaged every 5-7 days when the spheres reached a diameter of $100 \mu \mathrm{m}$.

Western blot assay of stemness markers. The normal CaSki cells and the CaSki tumor-forming cells were lysated with radioimmunoprecipitation assay buffer (catalogue no. P0013C; Beyotime Institute of Biotechnology, Haimen, China). Protein concentrations were determined using the Bio-Rad Protein assay (NanoDrop 2000c; Bio-Rad Laboratories Inc., Hercules, CA, USA) and $40 \mu \mathrm{g}$ protein was loaded per lane. Western blot analysis was performed according to standard procedures. Polyvinylidene difluoride (PVDF; EMD Millipore, Billerica, MA, USA) and 10\% sodium dodecyl sulfate polyacrylamide gel (Applygen Technologies Inc., Beijing, China) membranes were used for protein electrophoresis and transfer. Rabbit anti-mouse polyclonal Sox2 (catalogue no. ab59776; Abcam) and Oct4 (catalogue no. ab18976) antibodies were diluted at 1:1,000 and incubated with the membrane overnight at $4^{\circ} \mathrm{C}$. The blotted membranes were visualized using the ImageQuant ${ }^{\mathrm{TM}}$ LAS $4000 \mathrm{mini}$ system (GE Healthcare Life Sciences, Chalfont, UK).
Grey-scale values of the bands were analyzed quantitatively by Image Quant TL software (GE Healthcare Life Sciences). The levels of the stemness indicators, Sox 2 and Oct4, the drug resistance marker, ABCG2, and the apoptosis marker, PARP, were determined.

Telomerase activity assay. The telomerase activity of the CaSki sphere-forming cells and the normal CaSki cells was measured using the TRAPeze RT Telomerase Detection kit (catalogue no. S7710; EMD Millipore). The experimental process was performed in accordance with the manufacturer's protocols. Reactions were set up in triplicate. Samples were subjected to the following cycling parameters using an Applied Biosystems 7300 Real-Time PCR system: 1 cycle of $30 \mathrm{~min}$ at $30^{\circ} \mathrm{C}$ (extension of telomerase substrate) and 1 cycle of $2 \mathrm{~min}$ at $95^{\circ} \mathrm{C}$, followed by 45 cycles of $15 \mathrm{sec}$ at $94^{\circ} \mathrm{C}, 1 \mathrm{~min}$ at $59^{\circ} \mathrm{C}$ and $30 \mathrm{sec}$ at $45^{\circ} \mathrm{C}$ (PCR amplification of extended telomerase substrate). The calculation of the products (amount of DNA amplification produced by whole protein containing telomerase per $\mathrm{mg} / \mathrm{min}$ ) was performed according to the formula described previously (13). This experiment was repeated twice.

Cell growth assay. To determine the difference in the drug resistance between the normal CaSki cells and the CaSki tumor-forming cells, cells planted in 96-well plates were treated with different concentrations $(0.5,1,2$ and $5 \mathrm{ng} / \mathrm{ml})$ of cisplatin. The 3-(4,5-dimethylthiazol-2-yl)-5-(3-carboxymethoxyphenyl)-2(4-sulfophenyl)-2H-tetrazolium (MTS) assay was used to detect the inhibition of cell proliferation subsequent to $72 \mathrm{~h}$ of incubation at $37^{\circ} \mathrm{C}$. The optical density values of each well were measured with a microplate reader (model no. 680; Bio-Rad Laboratories Inc.) at $490 \mathrm{~nm}$. The half maximal inhibitory concentration $\left(\mathrm{IC}_{50}\right)$ was calculated using the SPSS software version 19 (IBM SPSS, Armonk, NY, USA). This experiment was repeated twice.

Cell invasion assay. The cell invasion assay was performed using Transwell chambers (Corning Inc., Corning, NY, USA) coated with $50 \mu$ l Matrigel polycarbonate membrane, as described in the manufacturer's protocols. The CaSki tumor-forming cells and normal CaSki cells, which grew at the exponential growth phase, were resuspended in serum-free DMEM and stem cell culturing medium at a concentration of $5 \times 10^{5}$ cells $/ \mathrm{ml}$. The single-cell suspension was added into the upper chamber (200 $\mu \mathrm{l} /$ well) and the lower chambers were loaded with complete medium $\left(500 \mu \mathrm{l} /\right.$ well). After $10 \mathrm{~h}$ of incubation at $37^{\circ} \mathrm{C}$, the cells that did not penetrate through the polycarbonate membrane were removed with wet cotton swabs, while the cells that had moved to the other side of the polycarbonate membrane were stained with $0.2 \%$ crystal violet dye for $30 \mathrm{~min}$. Subsequently, the cell numbers from four randomly selected fields were counted using a microscope (Nikon SE; Nikon Corporation, Tokyo,Japan). This experiment was repeated twice.

Statistical analysis. All statistical analyses were performed using SPSS 19.0 statistical software. Statistically significant differences between groups were determined using an unpaired Student's t-test. $\mathrm{P}<0.05$ was considered to indicate a statistically significant difference. 


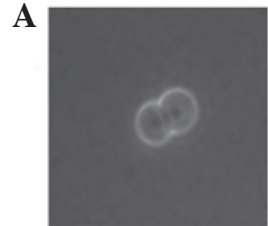

2 days

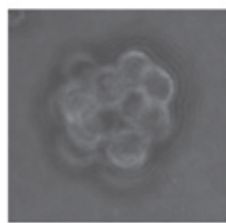

5 days

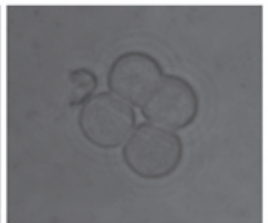

3 days

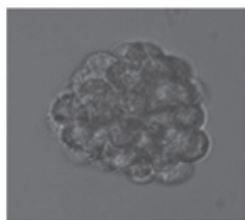

7 days

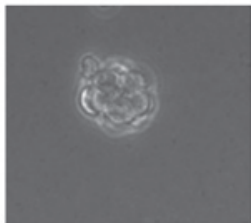

4 days

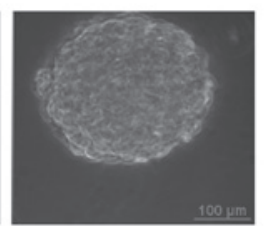

9 days
B

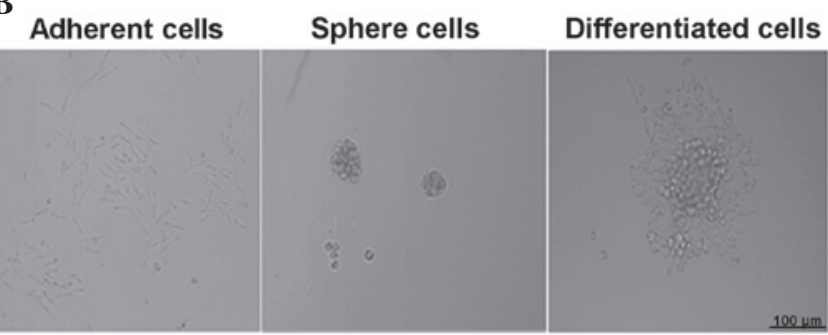

Figure 1. Tumor sphere formation and differentiation. (A) Generation of a sphere from one single cell (x200 magnification). (B) The tumor spheres were induced to differentiate following the addition of fetal bovine serum into the medium.
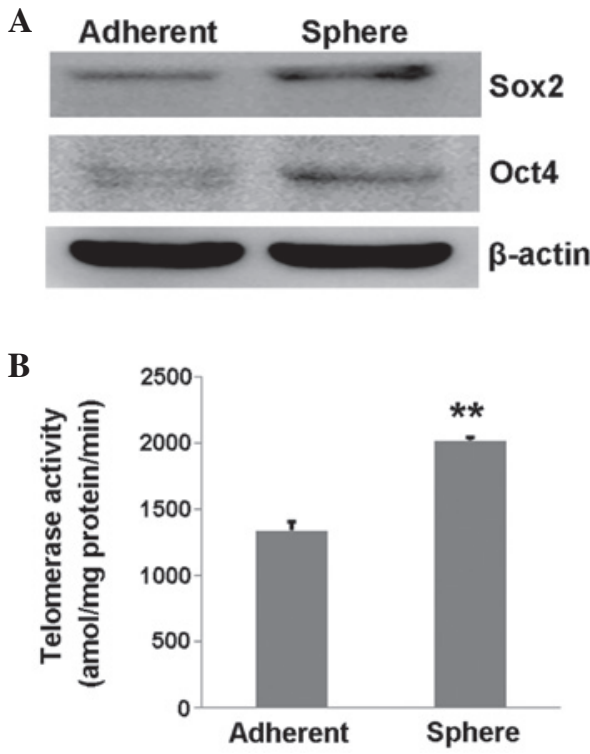

Figure 2. Stemness characteristics of tumor spheres. (A) Western blot analysis of the stemness-related proteins, Oct 4 and Sox2, in sphere-forming cells and tumor cells. (B) Histogram indicating the telomerase activity of the sphere-forming cells and the tumor cells. ${ }^{* *} \mathrm{P}<0.01 \mathrm{vs}$. control. Sox 2 , SRY-box 2; Oct4, POU class 5 homeobox 1.

\section{Results}

Cell culture of cervical CSCs. Cervical CSCs were selectively expanded using the stem cell medium. After 24-48 h, a proportion of the cells in stem cell medium were observed
A
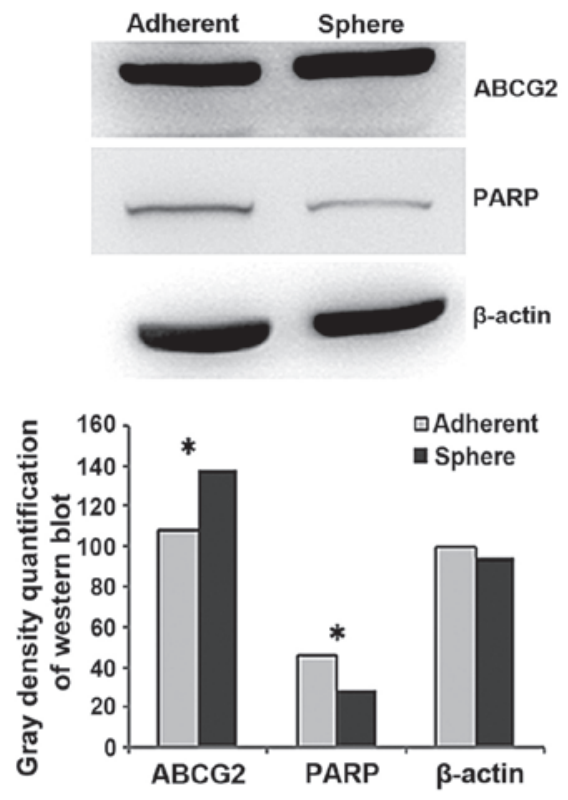

B

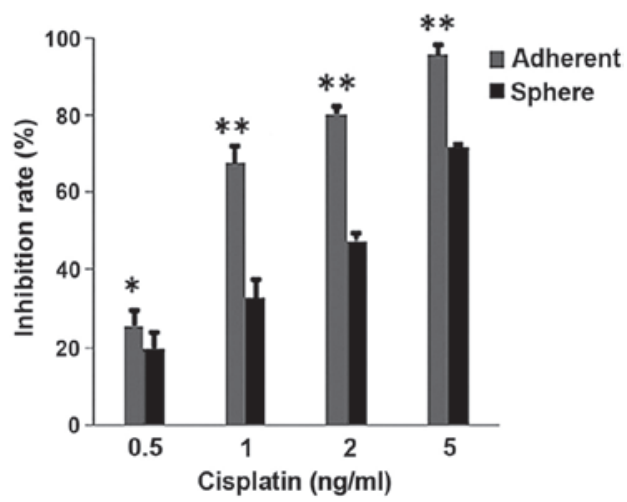

Figure 3. Drug resistance of tumor sphere cells. (A) Western bolt analysis of the drug resistance (ABCG2) and apoptosis-related (PARP) proteins in the sphere-forming cells and tumor cells. (B) 3-(4,5-dimethylthiazol-2-yl)5-(3-carboxymethoxyphenyl)-2-(4-sulfophenyl)-2H-tetrazolium growth inhibition analysis was used following cisplatin treatment in the sphere-forming cells and tumor cells. ABCG2, ATP binding cassette subfamily G member 2; PARP, poly (ADP-ribose) polymerase. ${ }^{*} \mathrm{P}<0.05,{ }^{* *} \mathrm{P}<0.01$.

to proliferate and grow into spheres. The bigger CaSki cell spheres $\sim 100 \mu \mathrm{m}$ in diameter were produced after 5-7 days of culturing and the rate of sphere formation was $0.15-0.2 \%$ (Fig. 1A). The cells in the spheres grew in a compacted pattern. When re-seeded in medium with FBS, the CaSki stem cell spheres were adherent to the bottom of the dish and started to differentiate (Fig. 1B).

Stemness of cervical cancer cells from spheres. First, the expression of stemness markers was tested using western blot and the expression of Oct 4 and Sox 2 was found to be increased in the CaSki sphere-forming cells compared with the normal control CaSki cells (Fig. 2A). Next, the telomerase activity was measured in a PCR-based assay that permitted precise quantitation of enzymatic activity with each prepared cell extract. The result showed that the telomerase activity in the CaSki sphere-forming cells was significantly higher than that in the control CaSki cells ( $\mathrm{P}=0.002$; Fig. $2 \mathrm{~B})$, suggesting that the CaSki sphere-forming cells had a higher proliferative ability. 
A

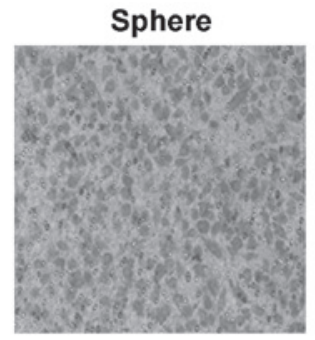

C

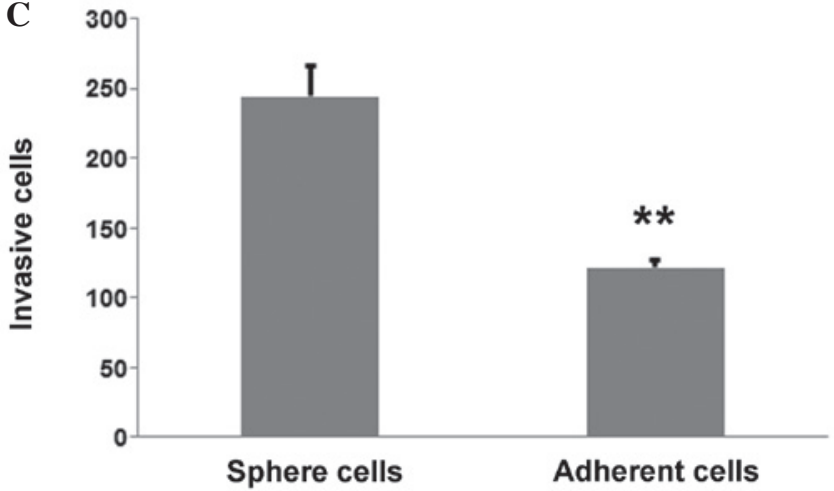

Figure 4. Invasive ability of tumor sphere cells. (A) Invasive cells of the adherent CaSki cells. (B) Invasive cells of the sphere-forming cells. (C) Histogram indicating that the CaSki sphere-forming cells were more invasive than the CaSki cells. ${ }^{* *} \mathrm{P}<0.01$ vs. control.

Drug resistance of cervical tumor sphere-forming cells. To demonstrate whether chemotherapeutics have a less suppressive effect on cervical CSCs than on cervical cancer cells, western blot and MTS assays were performed. The western blot results showed that the protein levels of ABCG2 and PARP were different between the CaSki sphere-forming cells and the CaSki cells (Fig. 3A). ABCG2, the drug transportation-related protein, was expressed at a higher level in the CaSki sphere-forming cells, while PARP, the apoptosis-related protein, was expressed at a lower level, when compared with the CaSki cells (Fig. 3A). The MTS results showed that cisplatin $(0.5,1,2$ and $5 \mathrm{ng} / \mathrm{ml})$ induced significantly less proliferation inhibition in the CaSki sphere-forming cells than in the CaSki cells $(\mathrm{P}=0.04,0.002$, 0.008 and 0.002 , respectively; Fig. $3 \mathrm{~B}$ ). The $\mathrm{IC}_{50}$ of cisplatin in the CaSki cells was $0.81 \mu \mathrm{g} / \mathrm{ml}$, while the $\mathrm{IC}^{50}$ in the CaSki sphere-forming cells was $2.06 \mu \mathrm{g} / \mathrm{ml}$. This line of evidence indicated that the CaSki sphere-forming cells are more resistant to chemotherapeutics than CaSki cells.

High invasive ability of cervical cancer cells. To investigate the difference in invasive ability between the CaSki sphere-forming cells and the CaSki cells, a Transwell assay was performed; the results showed that the CaSki sphere-forming cells (Fig. 4A) were more invasive than the CaSki cells (Fig. 4B). The number of invasive CaSki sphere-forming cells was $244 \pm 21.1$ compared with $121 \pm 5.5$ CaSki cells, which was determined to be statistically significant ( $\mathrm{P}=0.003$; Fig. 4C).

\section{Discussion}

Cervical cancer is a health threat to women that requires an urgent solution. Tumor relapse and drug resistance are the most troubling problems faced by patients and should be further illustrated mechanically to provide treatment strategies.

The present study performed selective enrichment of CaSki stem-like cells and conducted a telomerase activity assay, the results of which suggested that the CaSki sphere-forming cells not only highly expressed stemness markers, but also exhibited higher proliferative ability than CaSki cells. The MTS and cell invasion assays showed that the CaSki sphere-forming cells were more resistant to chemotherapy drugs (cisplatin) and more invasive than the CaSki cells.

Increasing evidence has highlighted the existence of CSCs and their role in tumor relapse and drug resistance $(14,15)$. The first successful isolation of CSCs from a solid tumor occurred in 2003 (16), demonstrating the existence of CSCs in cancers. In the present study, CaSki sphere-forming cells were isolated and shown to express the stemness markers, Oct4 and Sox2. It was also found that the CaSki sphere-forming cells exhibited higher telomerase activity than the CaSki cells. These results indicated the presence of CSCs in cervical cancer cells and their stronger proliferative ability than normal cells. Therefore, CSCs in cervical cancer may be the root cause of tumor recurrence and treatment failure (17).

After years of study on CSCs, it was found that serum-free medium with cytokines may be beneficial for the amplification of stem cells in vitro, maintaining the undifferentiated state and the potential of multi-directional differentiation. In the present study, it was shown that, following serum supplementation, the CaSki sphere-forming cells showed signs of differentiation. Therefore, this method is convenient for researchers to use to enrich the CSCs in the study of tumor phenotypes.

The drug resistance of tumor cells is the major cause of cancer treatment failure. The mechanisms of drug resistance involve, but are not limited to, the following aspects: The impairment of the DNA repair ability (18), the enrichment of P-glycoprotein (19), the glutathione transferase promotion of the metabolism of anticancer medicine (20) and the existence of CSCs (21). In the present study, the existence of CSCs in drug resistance was preliminarily evaluated. Highly-expressed stemness markers and the ability to form tumor spheres are considered as the hallmarks of CSCs, which are resistant to anticancer drugs. ABCG2, which is an efflux transporter on the cell membrane and a stem cell marker, is considered to confer drug resistance by expelling chemotherapeutic agents out of the cells (22). PARP, which is a DNA-repairing enzyme, is considered to be an important index of cell apoptosis and to play a crucial role DNA damage repair (23). In the present study, the expression of these two proteins was determined and the CaSki sphere-forming cells were shown to express a higher ABCG2 protein level and a lower PARP protein level than the CaSki cells. Although cisplatin is an effective anticancer drug in cervical cancer, it is unable to prevent cancer relapse. We hypothesized that CSCs may play a crucial role in drug resistance. Using the MTS assay, the difference in drug-resistance between the CaSki sphere-forming cells and CaSki cells was markedly illustrated, indicating that the CaSki sphere-forming cells were more resistant to cisplatin, 
demonstrating the stem cell characteristics as a potential mechanism for drug resistance.

Tumor metastasis, which is a fatal step in the progression of tumor disease, is a crucial sign for a poor prognosis. It has previously been suggested that CSCs may be at the center of this step (24). In the present study, in order to demonstrate the invasive ability of CaSki sphere-forming cells and CaSki cells, a Transwell invasion assay was performed, which found that the CaSki sphere-forming cells possessed higher invasive ability than the CaSki cells. This indicated that CSCs have a greater chance to metastasize than non-CSCs.

In conclusion, the present study demonstrated the presence of CSCs in cervical cancer that could be enriched by sphere-forming culturing. Under optimal conditions, the CaSki tumor spheres showed self-renewal, drug resistance and a strong invasion potential, which may lead to the recurrence of cervical cancer. This in vitro study described a suitable model for cervical cancer research on the mechanism of tumor relapse and metastasis.

\section{Acknowledgements}

This study was supported by grants from the National Natural Science Foundation of China (nos. 81071773, 30973447 and 81372158), the Natural Science Foundation of Beijing City (no. 7132071), the New Teacher Foundation for the Doctoral Program of Higher Education (no. 20101107120011), the Talents Project of Beijing (no. 2010D003034000043), the Open Issue of State Key Laboratory of Molecular Oncology (no. SKL-KF-2013-08) and the Independent Issue of State Key Laboratory of Molecular Oncology (no. SKL-2013-12).

\section{References}

1. Siegel R, Ma J, Zou Z and Jemal A: Cancer statistics, 2014. CA Cancer J Clin 64: 9-29, 2014.

2. Jemal A, Siegel R, Ward E, Murray T, Xu J and Thun MJ: Cancer statistics, 2007. CA Cancer J Clin 57: 43-66, 2007.

3. Leisching GR, Loos B, Botha MH and Engelbrecht AM: The role of mTOR during cisplatin treatment in an in vitro and ex vivo model of cervical cancer. Toxicology 335: 72-78, 2015.

4. Bonnet D and Dick JE: Human acute myeloid leukemia is organized as a hierarchy that originates from a primitive hematopoietic cell. Nat Med 3: 730-737, 1997.

5. Al-Hajj M, Wicha MS, Benito-Hernandez A, Morrison SJ and Clarke MF: Prospective identification of tumorigenic breast cancer cells. Proc Natl Acad Sci USA 100: 3983-3988, 2003.

6. Yan HC, Fang LS, Xu J, Qiu YY, Lin XM, Huang HX and Han QY: The identification of the biological characteristics of human ovarian cancer stem cells. Eur Rev Med Pharmacol Sci 18: 3497-3503, 2014

7. Yang CH, Wang HL, Lin YS, Kumar KP, Lin HC, Chang CJ, Lu CC, Huang TT, Martel J, Ojcius DM, et al: Identification of $\mathrm{CD} 24$ as a cancer stem cell marker in human nasopharyngeal carcinoma. PLoS One 9: e99412, 2014.

8. Chen YK, Huang AH and Lin LM: Sphere-forming-like cells (squamospheres) with cancer stem-like cell traits from VX2 rabbit buccal squamous cell carcinoma. Int J Oral Sci 6: 212-738, 2014.
9. Ueda K, Ogasawara S, Akiba J, Nakayama M, Todoroki K, Ueda K, Sanada S, Suekane S, Noguchi M and Matsuoka K: Aldehyde dehydrogenase 1 identifies cells with cancer stem cell-like properties in a human renal cell carcinoma cell line. PLoS One 8: e75463, 2013.

10. Batsaikhan BE, Yoshikawa K, Kurita N, Iwata T, Takasu C, Kashihara $\mathrm{H}$ and Shimada M: Cyclopamine decreased the expression of sonic hedgehog and its downstream genes in colon cancer stem cells. Anticancer Res 34: 6339-6344, 2014.

11. Peleg R, Romzova M, Kogan-Zviagin I, Apte RN and Priel E: Modification of topoisomerases in mammospheres derived from breast cancer cell line: Clinical implications for combined treatments with tyrosine kinase inhibitors. BMC Cancer 14: 910, 2014.

12. Sassi Fde A, Caesar L, Jaeger M, Nör C, Abujamra AL, Schwartsmann G, de Farias CB, Brunetto AL, Lopez PL and Roesler R: Inhibitory activities of trichostatin a in U87 glioblastoma cells and tumor sphere-derived cells. J Mol Neurosci 54: 27-40, 2014.

13. Benko AL, Olsen NJ and Kovacs WJ: Estrogen and telomerase in human peripheral blood mononuclear cells. Mol Cell Endocrinol 364: 83-88, 2012.

14. Castillo V, Valenzuela R, Huidobro C, Contreras HR and Castellon EA: Functional characteristics of cancer stem cells and their role in drug resistance of prostate cancer. Int J Oncol 45: 985-994, 2014.

15. Huang Y, Ju B, Tian J, Liu F, Yu H, Xiao H, Liu X, Liu W, Yao Z and Hao Q: Ovarian cancer stem cell-specific gene expression profiling and targeted drug prescreening. Oncol Rep 31: 1235-1248, 2014

16. Al-Hajj M, Wicha MS, Benito-Hernandez A, Morrison SJ and Clarke MF: Prospective identification of tumorigenic breast cancer cells. Proc Natl Acad Sci USA 100: 3983-3988, 2003.

17. Mannino M, Gomez-Roman N, Hochegger H and Chalmers AJ: Differential sensitivity of glioma stem cells to aurora kinase a inhibitors: Implications for stem cell mitosis and centrosome dynamics. Stem Cell Res 13: 135-143, 2014.

18. O'Grady S, Finn SP, Cuffe S, Richard DJ, O'Byrne KJ and Barr MP: The role of DNA repair pathways in cisplatin resistant lung cancer. Cancer Treat Rev 40: 1161-1170, 2014.

19. Chufan EE, Sim HM and Ambudkar SV: Molecular basis of the polyspecificity of P-glycoprotein (ABCB1): Recent biochemical and structural studies. Adv Cancer Res 125: 71-96, 2015.

20. Backos DS, Franklin CC and Reigan P: The role of glutathione in brain tumor drug resistance. Biochem Pharmacol 83: 1005-1012, 2012.

21. Kim JK, Jeon HY and Kim H: The molecular mechanisms underlying the therapeutic resistance of cancer stem cells. Arch Pharm Res 38: 389-401, 2015.

22. Zinzi L, Contino M, Cantore M, Capparelli E, Leopoldo M and Colabufo NA: ABC transporters in CSCs membranes as a novel target for treating tumor relapse. Front Pharmacol 5: 163, 2014.

23. Wielgos M and Yang ES: Discussion of PARP inhibitors in cancer therapy. Pharm Pat Anal 2: 755-766, 2013.

24. Chen T, Yang K, Yu J, Meng W, Yuan D, Bi F, Liu F, Liu J, Dai B and Chen X: Identification and expansion of cancer stem cells in tumor tissues and peripheral blood derived from gastric adenocarcinoma patients. Cell Res 22: 248-258, 2012. 